\title{
Association of serum uric acid levels with COVID-19 severity
}

Fang $\mathrm{Hu}^{1 \dagger}$, Yifan Guo ${ }^{1+}$, Jianghong Lin' ${ }^{1}$, Yingjuan Zeng ${ }^{1}$, Juan Wang ${ }^{2}$, Man $\mathrm{Li}^{3,4^{*}}$ and Li Cong ${ }^{1 *}$

\begin{abstract}
Aims: Hyperuricemia has attracted increasing attention. However, limited concern has been paid to the potential dangers of lowering serum uric acid (SUA). We observed lower levels of SUA in patients with COVID-19. Therefore, we aim to explore whether patients with COVID-19 had SUA lower than normal and the relationship of SUA and the severity of COVID-19.

Methods: This was a case-control study based on 91 cases with COVID-19 and 273 age- and sex-matched healthy control subjects. We first compared SUA levels and uric acid/creatinine (UA/Cr) ratio between patients with COVID19 and the healthy controls. Then, we examined the association of SUA levels and UA/Cr ratios with COVID-19 severity in COVID-19 cases only, defined according to the fifth edition of China's Diagnosis and Treatment Guidelines of COVID-19.
\end{abstract}

Results: SUA levels in patients with COVID-19 were 2.59\% lower, UA/Cr ratios 6.06\% lower at admission compared with healthy controls. In sex stratified analysis, levels of SUA and UA/Cr were lower in male patients with COVID-19 while only level of SUA was lower in female patients with COVID-19. Moreover, SUA and UA/Cr values were 4.27 and $8.23 \%$ lower in the severe group than that in the moderate group among male COVID-19 patients. Bivariate and partial correlations analysis showed negative correlations between SUA or UA/Cr ratio and COVID-19 after adjusting for age, sex, BMI and eGFR. A multiple linear regression analysis showed that SARS-CoV-2 infection and male sex were independent risk factors associated with lower SUA levels. Male patients with COVID-19 accompanied by low SUA levels had higher risk of developing severe symptoms than those with high SUA levels (incidence rate ratio: $4.05 ; 95 \% \mathrm{Cl}: 1.11,14.72$ ) at admission. Comparing SUA and $\mathrm{UA} / \mathrm{Cr}$ ratio at three time points (admission, discharge, and follow-up), we found that male patients experienced severe symptoms had lower SUA and $\mathrm{UA} / \mathrm{Cr}$ ratio levels comparing to moderate patients, but no significant difference between three time points. On the contrary, female patients had lower SUA and UA/Cr ratio at discharge than those at admission, but no significant difference of SUA and UA/Cr ratio between moderate and severe group.

(Continued on next page)

\footnotetext{
* Correspondence: liman26@mail.sysu.edu.cn; congli@mail.sysu.edu.cn

${ }^{\dagger}$ Fang Hu and Yifan Guo contributed equally to this work.

${ }^{3}$ Key Laboratory of Biomedical Imaging of Guangdong Province, Guangdong

Provincial Engineering Research Center of Molecular Imaging, The Fifth

Affiliated Hospital Sun Yat-sen University, Zhuhai, Guangdong, China

'Department of Endocrinology and Metabolism, The Fifth Affiliated Hospital

Sun Yat-Sen University, Zhuhai, Guangdong, China

Full list of author information is available at the end of the article
}

(c) The Author(s). 2021 Open Access This article is licensed under a Creative Commons Attribution 4.0 International License, which permits use, sharing, adaptation, distribution and reproduction in any medium or format, as long as you give appropriate credit to the original author(s) and the source, provide a link to the Creative Commons licence, and indicate if changes were made. The images or other third party material in this article are included in the article's Creative Commons licence, unless indicated otherwise in a credit line to the material. If material is not included in the article's Creative Commons licence and your intended use is not permitted by statutory regulation or exceeds the permitted use, you will need to obtain permission directly from the copyright holder. To view a copy of this licence, visit http://creativecommons.org/licenses/by/4.0/ The Creative Commons Public Domain Dedication waiver (http://creativecommons.org/publicdomain/zero/1.0/) applies to the data made available in this article, unless otherwise stated in a credit line to the data. 
(Continued from previous page)

Conclusion: Patients with COVID-19 had SUA and UA/Cr values lower than normal at admission. Male COVID-19 patients with low SUA levels had a significantly higher crude risk of developing severe symptoms than those with high SUA levels. During disease aggravation, the level of SUA gradually decreased until discharge. At the follow-up exam, the level of SUA was similar to the levels at admission.

Keywords: COVID-19, Uric acid, Uric acid/creatinine ratio

\section{Introduction}

COVID-19 has quickly spread throughout the world. By Mar 8, 2021, there were 116,363,935 cases worldwide and 2587,225 deaths. The mortality rate is as high as $2.22 \%$ [1]. However, the pathogenesis of COVID-19 is not clear, and there is currently no effective antiviral treatment. Therefore, it is very important to explore possible treatments according to its pathogenesis.

Although the infection pathways and pathogenesis of different viruses are not the same, the mechanisms by which they cause damage are similar [2]. Viral invasion causes an immune response, induces the activation of inflammatory factors, and causes the production of a large number of free radicals, including ROS (reactive oxygen species) and active nitrogen [3]. These free radicals produce oxidative stress, which can further activate the pathways of inflammatory factors. This cycle could enhance the immune response to eliminate the virus. However, more excessive immune response can also turn the defense mechanism into an injury pathway and aggravate the injury of the body [4]. Thus, oxidative stress plays a crucial role in viral invasion.

Serum uric acid (SUA) is the most abundant antioxidant molecule in the plasma. High SUA levels in humans represent an evolutionary advantage that can enhance antioxidant defense and prolong life [5]. Uric acid (UA) infusion into healthy volunteers increases SUA levels, which is associated with an increase in serum antioxidant capacity [6]. UA restores endothelial function in patients with type 1 diabetes and regular smokers via the antioxidants' stress response [7]. Therefore, the antioxidant effect of SUA may be potentially beneficial in situations characterized by oxidative stress, although the molecular mechanisms are not fully understood. SUA is thought to have a protective effect on both the central nervous system [8] and primary angleclosure glaucoma [9] against oxidative damage. However, there is a general agreement that hyperuricemia increases the risk of stroke and death [10], cardiovascular diseases [11], gout, insulin resistance, type 2 diabetes $[12,13]$, and all-cause mortality [14]. The higher mortality associated with more intense reductions in SUA are in line with the U-shaped association of SUA with mortality in some observational studies [15-18]. Hyperuricemia refers to $>420 \mu \mathrm{mol} / \mathrm{L}$ in men and $>360 \mu \mathrm{mol} / \mathrm{L}$ in women. Thus, it may be the most beneficial to control SUA within an appropriate range.

Some studies have investigated the relationship between SUA levels and inflammation (bacteria, viruses, or autoimmunity), but the conclusions were inconsistent $[8$, 19, 20]. Most studies showed that inflammation could induce the increase of SUA, particularly when the virus invaded the respiratory system [19]. However, SUA tends to decrease during a central nervous system infection $[8,20]$. Few studies have examined the association between SUA and COVID-19. SUA levels are clearly elevated in severely ill children compared with non-severely ill children on admission [21]. In our clinical work, we found that the levels of SUA in patients with COVID-19 were lower than average; hence, we aimed to explore the relationship between SUA and COVID-19 to better understand the pathophysiological process of COVID19.

\section{Methods}

\section{Data sources}

Our hospital, the Fifth Affiliated Hospital Sun Yat-sen University, is the only designated unit for the isolation treatment of COVID-19-diagnosed patients in Zhuhai city, Guangdong province. The study protocol was approved by the ethics committee of Fifth Affiliated Hospital Sun Yat-sen University (SYSU5). We did this study in accordance with the principles of the Declaration of Helsinki and Good Clinical Practice. A total of 364 subjects were studied, including 91 cases (there were 98 cases in our hospital during the period from January 17, 2020 to March 3, 2020, but 6 children were excluded. One patient with a high creatinine level and an estimated glomerular filtration rate (eGFR) of $<60 \mathrm{ml} /$ $\min ^{*} 1.73 \mathrm{~m}^{2}$ was excluded also, and 273 controls from the health management center in our hospital (matched 1:3 with the case group according to gender and age)). Because of the shortage of a healthy population with exact age matches, two 75-year-old female patients were paired with five 75-year-old women and a 74-year-old woman, and a 19-year-old female patient was paired with three 21-year-old female controls. The identification and classification of patients with COVID-19 was based on the criteria of the fifth edition of China's Diagnosis and Treatment Guidelines of COVID-19 [22]. 
Patients with COVID-19 were divided into mild, moderate, severe, and critically severe groups (Table S1). Due to a limited sample size, we grouped mild and moderate patients into the moderate group and severe and critically severe patients into the severe group. Nucleic acid tests were performed at Guangdong Center for Disease Control and Prevention. Complete laboratory data were available for both the control group and the case group. We recorded the patients' sex, age, disease history, laboratory examination, and treatments, with a particular focus on the SUA and creatinine levels at admission, discharge, and follow-up exams. Because renal function has an effect on SUA, we also used SUA/creatinine (UA/Cr) for statistical analysis. The most severe period was indicated by the lowest arterial partial pressure of oxygen $\left(\mathrm{PaO}_{2}\right)$ /fraction of inspiration oxygen $\left(\mathrm{FiO}_{2}\right)$. The Chronic Kidney Disease Epidemiology Collaboration (CKD-EPI) equation was used to calculate eGFR [23].

Fasting blood samples were collected from all patients after admission. Blood leukocyte (LEU), lymphocyte (LYM\%), fasting blood glucose (FBG), creatinine, UA, urea, albumin (ALB), globulin (GLB), lactate dehydrogenase (LDH), and a-hydroxybutyrate dehydrogenase $(\alpha-\mathrm{HBDH})$ were obtained in electric medical record system. Antiviral, anti-infective, and supportive treatments were used by the attending doctors according to the patients' conditions. COVID19 nucleic acid tests (throat swabs) were performed every other day. Patients whose nasal swabs were negative were considered cured and were discharged from the hospital.

\section{Statistical analysis}

The data were analyzed with SPSS 25.0 (SPSS Inc., Chicago, IL) and R (4.04). Normality was assessed with the Kolmogorov-Smirnoff test. The non-normal data were natural logarithm transformed to a normal distribution. The data that were normally distributed were represented by the mean \pm standard deviation (mean $\pm \mathrm{SD}$ ), and the means between two groups were compared using the independent Student's t-test. Non-normally distributed data were represented by the median and interquartile range [Md (P25-P75)], and the Mann-Whitney $U$ test was used to compare the medians between two groups. Categorical variables were expressed as the frequency (constituent ratio) $[\mathrm{n}(\%)]$. The rate or constituent ratio was compared with the Chi-squared test. The linear regression analysis was used to assess the relationships among the SUA levels, the UA/Cr ratio, and COVID19 outcome. The logistic regression analysis was used to assess the quantitative relationships among the SUA levels, the $\mathrm{UA} / \mathrm{Cr}$ ratio, and the severity of illness. Incidence rates were calculated as the number of events per person-days. Linear mixed-effects model was used to evaluate the change of the levels of UA and $\mathrm{UA} / \mathrm{Cr}$ ratio between moderate and severe patients at admission, discharge, and follow-up exams stratified by gender. $P<0.05$ was considered statistically significant.

\section{Results}

\section{Characteristics of the study subjects}

The age of the COVID-19 group was $47.53 \pm 15.43$ years old and that of the healthy control group was $47.55 \pm$ 15.33 years old (Table 1 ). There was no statistically significant difference in age and gender between two groups. The levels of FBG were higher in the case group than those in the controls $(P<0.001)$, whereas the eGFR, HGB, LEU, LYM\%, and ALB/GLB values were lower in the case group than those in the controls (Table 1). SUA levels were lower in the COVID-19 group than in the healthy controls $(P<0.001$ for overall; $P<0.001$ for males; $P=0.001$ for females). Serum UA/Cr ratios were also lower in the COVID-19 group than those in the healthy controls $(P=0.001$ for overall; $P=0.002$ for males; $P=0.07$ for females).

COVID-19 patients with severe symptoms were older, more likely to be male, and had a higher BMI, LYM\%, eGFR and ALB/GLB.Moreover $\mathrm{PaO} 2 / \mathrm{FiO} 2$ was lower in the severe group than that in the moderate group (Table 1). The AST, FBG, $\alpha-\mathrm{HBDH}$, and LDH levels were higher in the severe group than those in the moderate group (Table 1). The number of days from symptom appearance to hospitalization did not differ between the two groups. The days from admission to polymerase chain reaction (PCR) negative was longer in the severe group than that in the moderate group (11.50 (4.750-20.75) vs. 8.0 (4.0010.0), $P=0.04)$.

\section{Association of SARS-CoV-2 infection with SUA and UA/Cr}

Comparing to the age-, sex-matched healthy adults, patients with COVID-19 had lower UA and UA/Cr ratio (Fig. 1a, d) at baseline, despite whether they experienced severe symptoms. Furthermore, specifically in males, the UA level and $\mathrm{UA} / \mathrm{Cr}$ ratio were lower in severe patients than those in moderate patients $(P=0.002$ for $\mathrm{UA} ; P=0.046$ for $\mathrm{UA} / \mathrm{Cr}$ ratio; Fig. 1b, e). No statistically significant difference in either UA or $\mathrm{UA} / \mathrm{Cr}$ ratio was identified in female patients when comparing between severe and moderate groups (Fig. 1c, f). There was a negative correlation between SUA and COVID-19 outcome by either bivariate or partial correlation controlling by age, gender, BMI and $\operatorname{eGFR}(p<0.001)$. The same conclusion also could get between $\mathrm{UA} / \mathrm{Cr}$ and 
Table 1 Demographics and clinical characteristics of subjects

\begin{tabular}{|c|c|c|c|c|}
\hline \multirow[t]{2}{*}{ Factors } & \multirow{2}{*}{$\begin{array}{l}\text { Control } \\
\text { group } \\
(n=273)\end{array}$} & \multicolumn{3}{|l|}{ COVID-19 } \\
\hline & & Total $(n=91)$ & Moderate group $(n=69)$ & Severe group $(n=22)$ \\
\hline Age (years) & $47.55 \pm 15.33$ & $47.53 \pm 15.43$ & $44.00 \pm 14.32$ & $58.59 \pm 13.69^{\dagger, \neq}$ \\
\hline Gender (male/female) & $120 / 153$ & $40 / 51$ & $26 / 43$ & $14 / 8^{\ddagger}$ \\
\hline $\mathrm{BMI}\left(\mathrm{kg} / \mathrm{m}^{2}\right)$ & $23.58 \pm 3.38$ & $23.57 \pm 3.49$ & $23.18 \pm 3.41$ & $24.95 \pm 3.49^{\ddagger}$ \\
\hline $\mathrm{HGB}(\mathrm{g} / \mathrm{L})$ & $141.99 \pm 13.60$ & $138.23 \pm 17.39$ & $138.48 \pm 16.61$ & $137.45 \pm 20.34$ \\
\hline $\operatorname{LEU}^{*}\left({ }^{*} 10^{9} / \mathrm{L}\right)$ & $1.73 \pm 0.24$ & $1.59 \pm 0.32^{\dagger}$ & $1.59 \pm 0.32^{\dagger}$ & $1.58 \pm 0.32^{\dagger}$ \\
\hline LYM\% & $35.92 \pm 7.90$ & $32.54 \pm 10.46^{\dagger}$ & $34.17 \pm 9.72$ & $27.43 \pm 11.27^{\dagger, \neq}$ \\
\hline $\mathrm{eGFR}^{*}\left(\mathrm{ml} / \mathrm{min}^{*} 1.73 \mathrm{~m}^{2}\right)$ & $4.70 \pm 0.15$ & $4.66 \pm 0.15^{\dagger}$ & $4.68 \pm 0.15$ & $4.57 \pm 0.13^{\dagger, ~} \neq$ \\
\hline $\mathrm{AST}^{*}(\mathrm{U} / \mathrm{L})$ & $3.06 \pm 0.29$ & $3.03 \pm 0.39$ & $2.96 \pm 0.36$ & $3.24 \pm 0.41^{\dagger, \neq}$ \\
\hline ALB/GLB & $1.74 \pm 0.24$ & $1.35 \pm 0.22^{\dagger}$ & $1.38 \pm 0.22^{\dagger}$ & $1.24 \pm 0.18^{t, \neq}$ \\
\hline $\mathrm{a}-\mathrm{HBDH}^{*}(\mathrm{U} / \mathrm{L})$ & $4.91 \pm 0.17$ & $4.90 \pm 0.23$ & $4.84 \pm 0.20^{\dagger}$ & $5.06 \pm 0.24^{\dagger, \neq}$ \\
\hline $\mathrm{LDH}^{*}(\mathrm{U} / \mathrm{L})$ & $5.14 \pm 0.18$ & $5.14 \pm 0.25$ & $5.08 \pm 0.22^{\dagger}$ & $5.32 \pm 0.26^{\dagger, \neq}$ \\
\hline $\mathrm{FPG}(\mathrm{mmol} / \mathrm{L})$ & $4.66(4.39-5.05)$ & $5.31(4.890-6.18)^{\dagger}$ & $5.06(4.830-5.81)^{\dagger}$ & $5.80(5.340-7.36)^{\dagger, ~} \neq$ \\
\hline $\mathrm{PaO}_{2} / \mathrm{FiO}_{2}{ }^{*}$ & - & $5.94 \pm 0.53$ & $6.19 \pm 0.25$ & $5.12 \pm 0.36^{\ddagger}$ \\
\hline Days from onset to admission & - & $3.0(1.0-6.0)$ & $3.0(1.00-6.0)$ & $4.0(1.750-6.0)$ \\
\hline Days from admission to PCR negative) & - & $9.0(4.00-13.0)$ & $8.0(4.00-10.0)$ & $11.50(4.750-20.75)^{\ddagger}$ \\
\hline Diabetic patients (\%) & 0 & $9(9.9)$ & $4(5.8)$ & $5(22.7)^{\ddagger}$ \\
\hline Gout patients (\%) & 0 & 0 & & \\
\hline Hypertensive patients (\%) & 0 & $17(18.7)$ & $10(14.5)$ & $7(31.8)$ \\
\hline \multicolumn{5}{|l|}{$U A^{*}(\mu \mathrm{mol} / \mathrm{L})$} \\
\hline Total & $5.80 \pm 0.24$ & $5.65 \pm 0.28^{\dagger}$ & $5.66 \pm 0.29^{\dagger}$ & $5.62 \pm 0.25^{\dagger}$ \\
\hline Male & $5.95 \pm 0.18$ & $5.78 \pm 0.26^{\dagger}$ & $5.86 \pm 0.20^{\dagger}$ & $5.61 \pm 0.28^{\dagger, \neq}$ \\
\hline Female & $5.68 \pm 0.21$ & $5.55 \pm 0.26^{\dagger}$ & $5.53 \pm 0.26^{\dagger}$ & $5.63 \pm 0.20$ \\
\hline \multicolumn{5}{|l|}{ Creatinine $^{*}(\mu \mathrm{mol} / \mathrm{L})$} \\
\hline Total & $4.14 \pm 0.20$ & $4.10 \pm 0.26$ & $4.08 \pm 0.27$ & $4.16 \pm 0.22$ \\
\hline Male & $4.31 \pm 0.13$ & $4.30 \pm 0.21$ & $4.32 \pm 0.22$ & $4.26 \pm 0.19$ \\
\hline Female & $4.01 \pm 0.14$ & $3.94 \pm 0.18$ & $3.93 \pm 0.19^{\dagger}$ & $4.00 \pm 0.15$ \\
\hline \multicolumn{5}{|l|}{ UA/Cr ratio* } \\
\hline Total & $1.65 \pm 0.20$ & $1.55 \pm 0.27^{\dagger}$ & $1.58 \pm 0.24^{\dagger}$ & $1.45 \pm 0.32^{\dagger}$ \\
\hline Male & $1.64 \pm 0.20$ & $1.48 \pm 0.28^{\dagger}$ & $1.54 \pm 0.23^{\dagger}$ & $1.35 \pm 0.34^{\dagger, ~} \neq$ \\
\hline Female & $1.67 \pm 0.21$ & $1.60 \pm 0.24^{\dagger}$ & $1.60 \pm 0.25$ & $1.63 \pm 0.18$ \\
\hline
\end{tabular}

Abbreviations: $B M I$ Body mass index, HGB Hemoglobin, LEU Leukocyte, $L Y M$ Lymphocyte, eGFR Estimated glomerular filtration rate, AST Aspartic transaminase, $A L B$ Albumin, GLB Globulin, a-HBDH a-hydroxybutyrate dehydrogenase, $L D H$ Lactate dehydrogenase, $F P G$ Fasting plasma glucose, UA Uric acid, $C r C r e a t i n i n e$ Note: Data were expressed as the mean \pm standard deviation (SD) or median (P25-P75). Days (from onset to admission): the days from symptom appearance to hospitalization, days (from admission to PCR negative): the days from hospitalization to result of nucleic acid of SARS-CoV-2 negative $\mathrm{a}^{*}$ : The data were transformed into $\operatorname{Ln}(\mathrm{a})$

Compared with the control group: $P<0.05$ labeled as ${ }^{\dagger}$;

Compared with the moderate group: $P<0.05$ labeled as ${ }^{\ddagger}$

COVID-19 outcome (Table 2). By linear regression analysis, both SARS-CoV-2 infection and male gender were significantly associated with the UA levels after adjusting with other potential confounding factors including LEU, LYM\%, eGFR, ALB/GLB and FPG (Table 3). But, SARS-COV-2 infection was not an independent risk factor associated with the UA/
$\mathrm{Cr}$ levels after adjusting with other potential confounding factors including LEU, LYM\%, ALB/GLB and FPG $(P=0.07)$.

\section{Association with COVID-19 severity}

We grouped population to High-UA and Low-UA according to the levels of SUA median and gender. In 

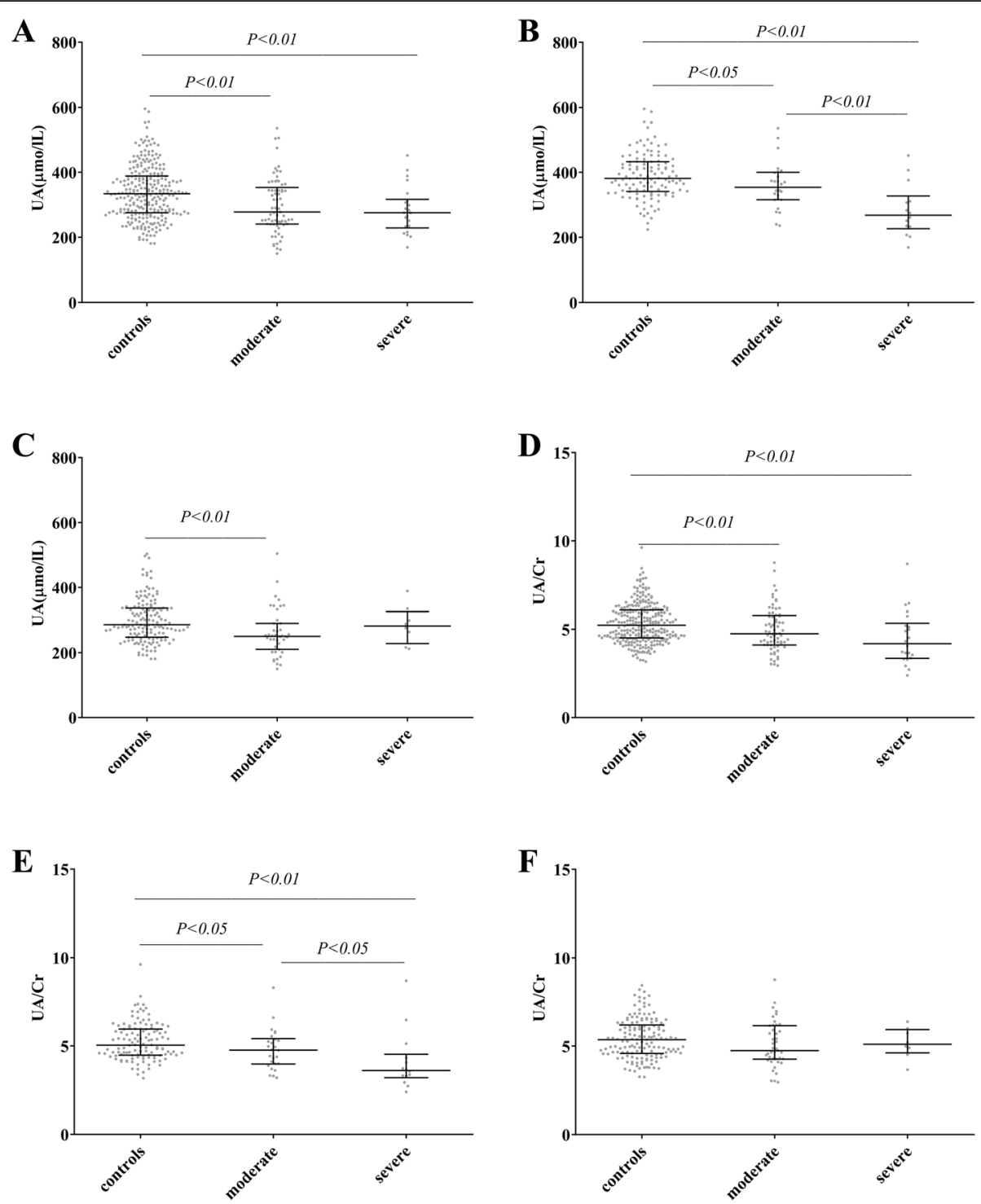

Fig. 1 Comparison of SUA and UA/Cr in patients with moderate, severe COVID-19 and control group. Note: Comparison of SUA and UA/Cr in patients with moderate, severe COVID-19 and control group: using Scatter dot plot. The line contained 50\% of all values (from 25th to 75th percentile) and was divided by the horizontal bar of the median value (50th percentile). (A) (D) Total subjects. (B)(E) Male subjects (C)(F) Female subjects

male patients with COVID-19, the incidence rate of developing severe symptoms was 4.05-fold higher (95\% CI: $1.11,14.72$ ) in low-UA group compared to the high-UA group. Nevertheless, there is no statistically significant difference in the incidence rate of developing severe symptoms when comparing between the low versus high-UA group in the female strata (incidence rate ratio 0.26; $95 \% \mathrm{CI}$ : 0.05, 1.29; Table 4).

Then, we assessed the correlation between $\mathrm{PaO} 2 /$ $\mathrm{FiO} 2$ and $\mathrm{UA}, \mathrm{UA} / \mathrm{Cr}$ ratio in male patients of

Table 2 Correlation between COVID-19 and UA*, UA/Cr ratio*

\begin{tabular}{|c|c|c|}
\hline & Bivariate correlation ( $P$ value) & partial correlation ( $P$ value $)$ \\
\hline $\mathrm{UA}^{*}$ & $-0.23(P<0.001)$ & $-0.34(P<0.001)$ \\
\hline UA/Cr ratio ${ }^{*}$ & $-0.19(P<0.001)$ & $-0.18(P=0.001)$ \\
\hline
\end{tabular}

Note: Partial correlation is controlling by age, gender, BMI and eGFR. Spearman test were used

Abbreviations: BMI Body mass index, eGFR Estimated glomerular filtration rate, UA Uric acid, $\mathrm{Cr}$ Creatinine

$a^{*}:$ The data were transformed into $\operatorname{Ln}(a)$ 
Table 3 Association of UA*, UA/Cr ratio ${ }^{*}$ with COVID-19 and gender

\begin{tabular}{|c|c|c|c|c|}
\hline & & Model 1 & Model 2 & Model 3 \\
\hline & & $\beta(95 \% \mathrm{Cl})$ & $\beta(95 \% \mathrm{Cl})$ & $\beta(95 \% \mathrm{Cl})$ \\
\hline$\overline{U A^{\#}}$ & COVID-19 & $-0.25^{\dagger}(-0.20,-0.10)$ & $-0.21^{\dagger}(-0.20,-0.06)$ & $-0.15^{\dagger}(-0.17,-0.01)$ \\
\hline & Male & $0.50^{\dagger}(0.22,0.31)$ & $0.53^{\dagger}(0.22,0.33)$ & $0.47^{\dagger}(0.19,0.30)$ \\
\hline & COVID-19*Male & - & $-0.06(-0.15,0.06)$ & $-0.001(-0.10,0.10)$ \\
\hline & AlC & -79.27 & -78.03 & -111.46 \\
\hline & $\mathrm{BIC}$ & -63.68 & -58.54 & -72.49 \\
\hline UA/Cr ratio ${ }^{\#}$ & COVID-19 & $-0.21^{\dagger}(-0.16,-0.06)$ & $-0.13(-0.13,0.01)$ & $-0.06(-0.11,0.05)$ \\
\hline & Male & $-0.12^{\dagger}(-0.10,-0.01)$ & $-0.07(-0.09,0.02)$ & $-0.08(-0.09,-0.02)$ \\
\hline & COVID-19*Male & - & $-0.13(-0.20,-0.01)$ & $-0.11(-0.18,0.02)$ \\
\hline & AIC & -68.02 & -69.28 & -83.80 \\
\hline & $\mathrm{BIC}$ & -52.43 & -49.80 & -48.72 \\
\hline
\end{tabular}

Note: Linear regression analysis was used to assess association of UA*, UA/Cr ratio* with COVID-19 and gender

Abbreviations: AIC Akaike information criterion, BIC Bayesian Information Criterions

Model 1: Including COVID-19 and gender

Model 2: Including Model 1 and COVID-19*gender

Model 3 of UA* : Including Model 2 and LEU ${ }^{\# \dagger}, \mathrm{LYM}^{\dagger}{ }^{\dagger}$, eGFR ${ }^{\# \dagger}, \mathrm{ALB} / \mathrm{GLB}, \mathrm{FPG}$

Model 3 of UA/Cr ratio ${ }^{\#}$ : Including Model 2 and LEU ${ }^{\#+}$, LYM ${ }^{\dagger}{ }^{+}, A L B / G L B, F P G$

The $P$-values are for the beta coefficient

$a^{\#}$ : The data were transformed into $\operatorname{Ln}(\mathrm{a})$

${ }^{\dagger} P<0.05$

COVID-19 by Pearson test. PaO2/FiO2, as an important respiratory parameter, could roughly reflect the severity of illness. As shown in Table 5, there was positive correlation between SUA and $\mathrm{PaO} 2 /$ FiO2 by either bivariate or partial correlation controlling by age, BMI and $\operatorname{eGFR}(p<0.05)$. But there was no association between $\mathrm{UA} / \mathrm{Cr}$ and $\mathrm{PaO} 2 / \mathrm{FiO} 2$ by partial correlation controlling by age, BMI and eGFR $(p=0.058)$.

As shown in Table 6, the univariate analysis revealed that both $\mathrm{UA}$ and $\mathrm{UA} / \mathrm{Cr}$ were associated with the severity of COVID-19 (OR 0.01; 95\% CI: 0.00, $0.30 ; P=0.008$ for UA OR $0.07 ; 95 \%$ CI: $0.004,1.07$; $P=0.01$ for $\mathrm{UA} / \mathrm{CR})$. However, these associations did not remain statistically significant after adjusting for other potential confounding factors. SUA and UA/Cr on admission were not independent risk factors for the severity of COVID-19.

\section{Longitudinal effect of the virus on SUA}

We fitted linear mixed-effects models with random intercept and slope for time to investigate whether changes in UA and $\mathrm{UA} / \mathrm{Cr}$ ratio over time were associated with the severity level of COVID-19 symptoms when stratified by gender. In males, patients with severe symptoms had significantly lower SUA and UACR levels comparing to moderate patients (SUA effect size $-0.17,95 \%$ CI $-0.29,-0.05$; UACR effect

Table 4 Incidence rate ratio of COVID-19 patients

\begin{tabular}{|c|c|c|c|c|c|}
\hline & UA Group & $\begin{array}{l}\text { Severe } \\
\text { COVID-19 } \\
\end{array}$ & Person-Days & Incidence Rate & Incidence Rate Ratio $(95 \% \mathrm{Cl})$ \\
\hline \multirow[t]{2}{*}{ Total } & High-UA $(n=45)$ & $10(22.2 \%)$ & 323 & 0.031 & $0.84(0.36,1.98)$ \\
\hline & Low-UA $(n=45)$ & $11(24.4 \%)$ & 423 & 0.026 & \\
\hline \multirow[t]{2}{*}{ Male } & High-UA $(n=19)$ & $3(15.8 \%)$ & 152 & 0.020 & $4.05(1.11,14.72)$ \\
\hline & Low-UA $(n=20)$ & $10(55.0 \%)$ & 123 & 0.081 & \\
\hline \multirow[t]{2}{*}{ Female } & High-UA $(n=25)$ & $6(24.0 \%)$ & 257 & 0.023 & $0.26(0.05,1.29)$ \\
\hline & Low-UA $(n=26)$ & $2(7.7 \%)$ & 313 & 0.006 & \\
\hline
\end{tabular}

Notes: the cut-off point of total patients between high-UA and low-UA is $277 \mu \mathrm{mol} / \mathrm{L}$ (Median)

the cut-off point of male patients between high-UA and low-UA is $334 \mu \mathrm{mol} / \mathrm{L}$ (Median)

the cut-off point of female patients between high-UA and low-UA is $252 \mu \mathrm{mol} / \mathrm{L}$ (Median)

Days referred to the time from admission to the severe period

One patient was not analyzed because he was severe type at admission 
Table 5 Correlation between $\mathrm{PaO}_{2} / \mathrm{FiO}_{2}{ }^{*}$ and $\mathrm{UA}^{*}$, UA/Cr ratio ${ }^{*}$ in male patients of COVID-19

\begin{tabular}{lll}
\hline & Bivariate correlation $(P$ value $)$ & partial correlation $(P$ value $)$ \\
\hline $\mathrm{UA}^{*}$ & $0.463(P=0.003)$ & $0.411(P=0.013)$ \\
$\mathrm{UA}^{*} \mathrm{Cr}$ ratio & \\
\hline
\end{tabular}

Note: Partial correlation is controlling by age, BMI and eGFR. Pearson test were used

Abbreviations: BMI Body mass index, eGFR Estimated glomerular filtration rate, UA Uric acid, $C r$ creatinine

$a^{*}:$ The data were transformed into $\operatorname{Ln}(a)$

size $-0.19,95 \%$ CI $-0.35,-0.04)$, however we did not observe significant difference between different time points (Table 7). In females, we found no statistical difference of either SUA or UACR levels between severe patients and moderate patients. Nevertheless, female patients have lower SUA and UACR levels at discharge comparing to their levels at admission (SUA effect size $-0.11,95 \%$ CI $-0.18,-0.04$; UACR effect size $-0.18,95 \%$ CI $-0.25,-0.12$ ). At first follow-up exam, these differences disappeared (Fig. 2).

\section{Discussion}

Our study showed that SUA and UA/Cr levels at admission were lower in patients with COVID-19 than controls, particularly in males. Moreover, SUA and UA/Cr values were lower in the severe group than in the moderate group among male patients with COVID-19. Male patients with COVID-19 with low SUA levels at had a higher risk of developing severe symptoms than those with high SUA levels. There was a positive correlation between SUA and $\mathrm{PaO}_{2} / \mathrm{FiO}_{2}$. During disease aggravation, the level of SUA gradually decreased until discharge. Hence, SUA is closely related to the severity of COVID-19, although it is not an independent risk factor, regardless of whether low SUA is the cause or result of the illness.

SUA is a powerful antioxidant that accounts for over half of the free radical scavenging activity in human blood by reducing superoxide and singlet oxygen and protecting the oxidation of vitamin $\mathrm{C}$ through the chelation of iron [24]. Since neurons are highly susceptible to oxidative stress, decreased SUA levels are present in central nervous system disorders such as Alzheimer's disease [25], GuillainBarre syndrome and many types of meningitis (viral meningitis or meningoencephalitis, brain cysticercosis, tuberculous meningitis or meningoencephalitis, cryptococcus meningitis or meningoencephalitis, and bacterial meningitis or meningoencephalitis) [26]. Conversely, SUA levels are increased in infections of other systems. Respiratory syncytial virus (RSV) induces increased UA levels in mouse neonates, and the inhibition of UA by xanthine oxidase inhibitor decreases mucus production, reduces cellular infiltrates to the lungs (particularly ILC2s), and decreases type 2 immune responses [19]. UA is a biomarker of early cystic fibrosis lung disease [27], and high SUA is positively correlated with severe infections such as sepsis [28]. Hence, it is important to monitor changes of SUA level in infectious diseases.

In our clinical observation, patients with COVID-19 infection had lower SUA levels than the normal range. It is consistent with previous research findings $[29,30]$. Severely infected patients had lower SUA levels, and this trend was more obvious in men. However, the mechanism was unclear. First, as a primary antioxidant, SUA could be consumed by oxidizing

Table 6 Linear mixed effects models of changes in the levels of UA and UA/Cr ratio between severe and moderate symptoms and three time points by gender

\begin{tabular}{|c|c|c|c|c|c|c|c|c|}
\hline \multirow{3}{*}{$\begin{array}{l}\text { Fixed } \\
\text { Effects }\end{array}$} & \multicolumn{4}{|l|}{ UA } & \multicolumn{4}{|l|}{ UA/Cr ratio } \\
\hline & \multicolumn{2}{|l|}{ Male } & \multicolumn{2}{|l|}{ Female } & \multicolumn{2}{|l|}{ Male } & \multicolumn{2}{|l|}{ Female } \\
\hline & Effect Size & $95 \% \mathrm{Cl}$ & Effect Size & $95 \% \mathrm{Cl}$ & Effect Size & $95 \% \mathrm{Cl}$ & Effect Size & $95 \% \mathrm{Cl}$ \\
\hline Moderate & Ref & Ref & Ref & Ref & Ref & Ref & Ref & Ref \\
\hline Severe & -0.17 & $(-0.29,-0.05)$ & 0.07 & $(-0.11,0.24)$ & -0.19 & $(-0.35,-0.04)$ & 0.02 & $(-0.15,0.19)$ \\
\hline Admission & Ref & Ref & Ref & Ref & Ref & Ref & Ref & Ref \\
\hline Discharge & -0.05 & $(-0.12,0.01)$ & -0.11 & $(-0.18,-0.04)$ & -0.06 & $(-0.14,0.01)$ & -0.18 & $(-0.25,-0.12)$ \\
\hline Follow-up & 0.04 & $(-0.04,0.13)$ & 0.04 & $(-0.03,0.11)$ & 0.05 & $(-0.04,0.14)$ & -0.03 & $(-0.10,0.04)$ \\
\hline
\end{tabular}


Table 7 Levels of UA and UA/Cr ratio between severe and moderate symptoms and three time points by gender

\begin{tabular}{|c|c|c|c|c|c|c|c|c|}
\hline \multirow{3}{*}{$\begin{array}{l}\text { Fixed } \\
\text { Effects }\end{array}$} & \multicolumn{4}{|l|}{ UA } & \multicolumn{4}{|l|}{ UA/Cr ratio } \\
\hline & \multicolumn{2}{|l|}{ Male } & \multicolumn{2}{|l|}{ Female } & \multicolumn{2}{|l|}{ Male } & \multicolumn{2}{|l|}{ Female } \\
\hline & Effect Size & $95 \% \mathrm{Cl}$ & Effect Size & $95 \% \mathrm{Cl}$ & Effect Size & $95 \% \mathrm{Cl}$ & Effect Size & $95 \% \mathrm{Cl}$ \\
\hline Moderate & Ref & Ref & Ref & Ref & Ref & Ref & Ref & Ref \\
\hline Severe & -0.17 & $(-0.29,-0.05)$ & 0.07 & $(-0.11,0.24)$ & -0.19 & $(-0.35,-0.04)$ & 0.02 & $(-0.15,0.19)$ \\
\hline Admission & Ref & Ref & Ref & Ref & Ref & Ref & Ref & Ref \\
\hline Discharge & -0.05 & $(-0.12,0.01)$ & -0.11 & $(-0.18,-0.04)$ & -0.06 & $(-0.14,0.01)$ & -0.18 & $(-0.25,-0.12)$ \\
\hline Follow-up & 0.04 & $(-0.04,0.13)$ & 0.04 & $(-0.03,0.11)$ & 0.05 & $(-0.04,0.14)$ & -0.03 & $(-0.10,0.04)$ \\
\hline
\end{tabular}

Note: Linear mixed effects models were used to assess changes of levels of UA and UA/Cr ratio between severe and moderate symptoms and three time points by gender

Abbreviations: UA Uric acid, $\mathrm{Cr}$ Creatinine

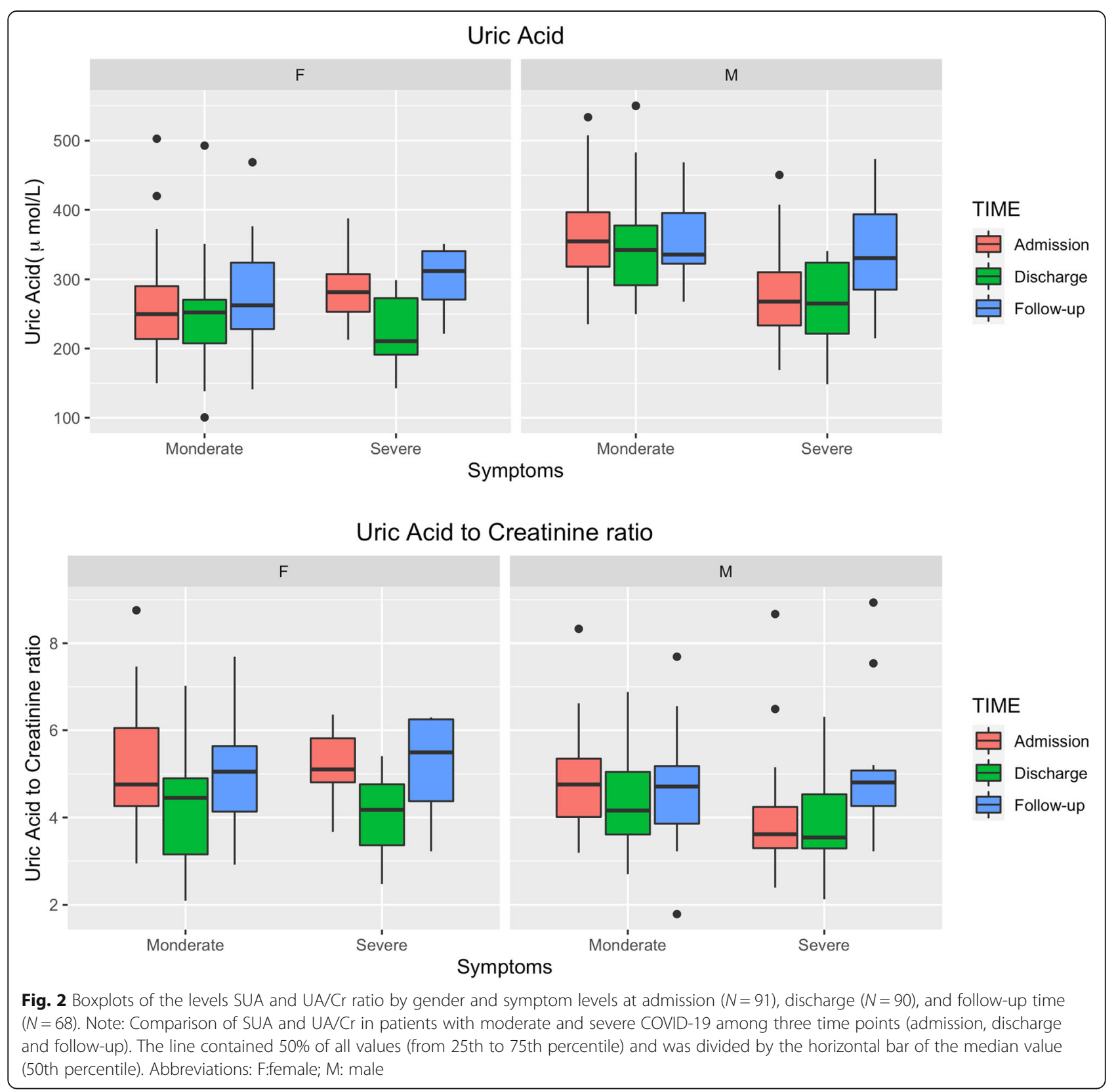


agents to prevent an inflammatory response. Therefore, systemic inflammation and oxidative stress were likely to cause an obvious consumption of UA and a significant decrease in its serum levels. We suggested that the decreased SUA levels may play a part in the anti-oxidative insufficiency, which could contribute to COVID-19 development. Second, serum metabolomic analysis of patients with COVID-19 showed that guanosine monophosphate (GMP) levels were lower in patients with COVID-19 than in healthy people. In addition, GMP levels were lower in the severe group compared with the moderate group [31]. GMP is eventually metabolized into SUA and excreted out of the body. Thus, SUA decreases following decreased GMP levels in patients with COVID-19. Finally, CD39 and CD73 increase because of inflammation. Increased CD39 and CD73 could break adenosine triphosphate (ATP) down into adenosine monophosphate (AMP) and AMP down into adenosine. Hence, we speculated AMP was decreased in patients with COVID-19. While AMP is a raw material of SUA, SUA was reduced in patients with COVID19 following [32]. Finally, low SUA might occur due to a specific dysfunction of the kidney proximal tubule caused by COVID-19 [30].

We found that patients with low SUA and UA/Cr levels at admission had a higher incidence rate of developing severe symptoms of COVID-19 later. However, SUA levels and UA/Cr were not the independent risk factors of developing severe disease. Thus, the deterioration of the disease may be the result of the joint action of multiple factors. It is known that hyperuricemia increases the risk of stroke and death, cardiovascular diseases, and gout. Moreover, hypouricemia is now recognized to increase adverse disease outcome; however, the cutoff is difficult to determine. Determining the roles of SUA and oxidative stress in COVID-19 is quite difficult. The mechanism of SUA in the pathogenesis of COVID-19 is should be further explored in a future study with a larger sample size. Increasing SUA levels may be a potential COVID-19 treatment method.

Our study also noted that the relationship between SUA, UA/Cr, and COVID-19 was more obvious in the male population. There were no relevant previous studies reporting this finding. SUA levels were higher in men than in women among healthy people. Testosterone might upregulate the expression of the urater transporter 1 gene, thereby increasing the reabsorption of UA and the level of SUA [33]. In addition, SUA had different effects on the incidence of thyroid nodules [34] and fat distribution [35] in different genders. We speculated that the male oxidative stress response was stronger than the female response, so more SUA must be consumed in males. Thus, SUA might play a more important role in oxidative stress in males.

In addition, previous research showed that SUA levels were clearly elevated in severely ill children compared with non-severely ill children on admission. Our study population was adults; hence, the conclusion was opposite. Confirmation of changes in SUA levels with infectious disease in different age groups requires a future study with a larger sample size.

Our data also showed that although patients in both the severe and moderate groups had met the hospital discharge criteria in which they were required to have two consecutive negative COVID-19 nucleic acid tests, their SUA levels deceased upon discharge in female patients, which suggests that these discharged patients had not fully recovered physiologically from the impacts of COVID-19. These patients require further strengthening, nutritional support, and rest. In addition, we found that SUA and $\mathrm{UA} / \mathrm{Cr}$ played the same role in predicting the severity of the disease in patients with COVID-19 with a normal level of creatinine.

We acknowledge that our present study has some limitations. First, the patients with COVID-19 were divided into mild, moderate, severe, and most severe according to the fifth edition of China's Diagnosis and Treatment Guidelines of COVID-19. We grouped the mild and moderate patients in the moderate group and the severe and most severe patients in the severe group because of the limited number of patients. Second, it was uncertain whether low SUA levels can contribute to a higher risk of COVID-19 infection because of the lack of SUA level data prior to admission. Third, the relationship between the change of SUA and the risk to severe disease was uncertain because of the lack of regular SUA examinations.

The present study demonstrated that SUA levels and the UA/Cr ratio were decreased and negatively associated with COVID-19 severity, which suggests a possible association between SUA levels with the development of COVID-19 and the involvement of oxidative stress in the pathogenesis of COVID-19.

\footnotetext{
Abbreviations

SUA: Serum uric acid; COVID-19: Coronavirus disease; Cr: creatinine; SARSCoV-2: Severe acute respiratory syndrome coronavirus 2; ROS: Reactive oxygen species; UA: Uric acid; SYSU5: Fifth Affiliated Hospital Sun Yat-sen University; eGFR: Estimated glomerular filtration rate; $\mathrm{PaO}_{2}$ : Partial pressure of oxygen; $\mathrm{FiO}_{2}$ : Fraction of inspiration oxygen; CKD-EPI: Chronic kidney disease epidemiology collaboration; LEU: Leukocyte; NEU: Neutrophil; FBG: Fasting blood glucose; a-HBDH: a-hydroxybutyrate dehydrogenase; HGB: Hemoglobin; LYM: Lymphocyte; BMI: Body mass index; PCR: Polymerase
} chain reaction; RSV: Respiratory syncytial virus; GMP: Guanosine 


\section{Appendix}

\section{Statement of guideline}

Table 8 STROBE Statement-Checklist of items that should be included in reports of case-control studies

\begin{tabular}{|c|c|c|c|}
\hline & $\begin{array}{l}\text { Item } \\
\text { No }\end{array}$ & Recommendation & $\begin{array}{l}\text { Page } \\
\text { No }\end{array}$ \\
\hline \multirow[t]{2}{*}{ Title and abstract } & 1 & (a) Indicate the study's design with a commonly used term in the title or the abstract & 1 \\
\hline & & (b) Provide in the abstract an informative and balanced summary of what was done and what was found & $5-7$ \\
\hline \multicolumn{4}{|l|}{ Introduction } \\
\hline $\begin{array}{l}\text { Background/ } \\
\text { rationale }\end{array}$ & 2 & Explain the scientific background and rationale for the investigation being reported & $8-9$ \\
\hline Objectives & 3 & State specific objectives, including any prespecified hypotheses & $9-10$ \\
\hline \multicolumn{4}{|l|}{ Methods } \\
\hline Study design & 4 & Present key elements of study design early in the paper & $10-12$ \\
\hline Setting & 5 & $\begin{array}{l}\text { Describe the setting, locations, and relevant dates, including periods of recruitment, exposure, follow-up, and } \\
\text { data collection }\end{array}$ & $10-12$ \\
\hline \multirow[t]{2}{*}{ Participants } & 6 & $\begin{array}{l}\text { (a) Give the eligibility criteria, and the sources and methods of case ascertainment and control selection. } \\
\text { Give the rationale for the choice of cases and controls }\end{array}$ & $10-11$ \\
\hline & & (b) For matched studies, give matching criteria and the number of controls per case & $10-11$ \\
\hline Variables & 7 & $\begin{array}{l}\text { Clearly define all outcomes, exposures, predictors, potential confounders, and effect modifiers. Give } \\
\text { diagnostic criteria, if applicable }\end{array}$ & $10-12$ \\
\hline $\begin{array}{l}\text { Data sources/ } \\
\text { measurement }\end{array}$ & $8^{*}$ & $\begin{array}{l}\text { For each variable of interest, give sources of data and details of methods of assessment (measurement). } \\
\text { Describe comparability of assessment methods if there is more than one group }\end{array}$ & $10-12$ \\
\hline Bias & 9 & Describe any efforts to address potential sources of bias & $10-12$ \\
\hline Study size & 10 & Explain how the study size was arrived at & $10-11$ \\
\hline $\begin{array}{l}\text { Quantitative } \\
\text { variables }\end{array}$ & 11 & $\begin{array}{l}\text { Explain how quantitative variables were handled in the analyses. If applicable, describe which groupings } \\
\text { were chosen and why }\end{array}$ & $11-12$ \\
\hline \multirow[t]{5}{*}{ Statistical methods } & 12 & (a) Describe all statistical methods, including those used to control for confounding & $12-13$ \\
\hline & & (b) Describe any methods used to examine subgroups and interactions & $12-13$ \\
\hline & & (c) Explain how missing data were addressed & 10 \\
\hline & & (d) If applicable, explain how matching of cases and controls was addressed & $10-11$ \\
\hline & & (e) Describe any sensitivity analyses & $12-13$ \\
\hline
\end{tabular}

\section{Results}

Participants

(a) Report numbers of individuals at each stage of study —eg numbers potentially eligible, examined for eligibility, confirmed eligible, included in the study, completing follow-up, and analysed

(b) Give reasons for non-participation at each stage

(c) Consider use of a flow diagram

(a) Give characteristics of study participants (eg demographic, clinical, social) and information on exposures and potential confounders

(b) Indicate number of participants with missing data for each variable of interest

Outcome data

Main results

Other analyses

\section{Discussion}

Key results

Limitations

(a) Give unadjusted estimates and, if applicable, confounder-adjusted estimates and their precision (eg, 95\% confidence interval). Make clear which confounders were adjusted for and why they were included

(b) Report category boundaries when continuous variables were categorized

(c) If relevant, consider translating estimates of relative risk into absolute risk for a meaningful time period Report other analyses done_-eg analyses of subgroups and interactions, and sensitivity analyses 
Table 8 STROBE Statement—Checklist of items that should be included in reports of case-control studies (Continued)

\begin{tabular}{lcl}
\hline & $\begin{array}{c}\text { Item } \\
\text { No }\end{array}$ & Recommendation \\
\hline $\begin{array}{l}\text { Generalisability } \\
\text { Other information } \\
\text { Funding }\end{array}$ & 21 & $\begin{array}{l}\text { results from similar studies, and other relevant evidence } \\
\text { Discuss the generalisability (external validity) of the study results } \\
\text { No }\end{array}$ \\
\hline & 22 & $\begin{array}{l}\text { Give the source of funding and the role of the funders for the present study and, if applicable, for the } \\
\text { original study on which the present article is based }\end{array}$ \\
23
\end{tabular}

monophosphate; ATP: Adenosine triphosphate; AMP: Adenosine monophosphate

\section{Supplementary Information}

The online version contains supplementary material available at https://doi. org/10.1186/s12902-021-00745-2.

Additional file 1: Table 1S Clinical classification of the COVID-19.

\section{Acknowledgements}

Not applicable.

\section{Authors' contributions}

Study concept and design: Fang Hu, Li Cong, and Man Li. Acquisition of data: Fang Hu, Yifan Guo, Jianghong Lin, Yingjuan Zeng, Li Cong, and Juan Wang. Analysis of data: Yifan Guo, Man Li, and Fang Hu. Drafting of the manuscript: Fang Hu. Critical revision of the manuscript for important intellectual content: Li Cong and Man Li. All authors contributed to the manuscript for important intellectual content and approved the submission, as well as read and approved the final manuscript.

\section{Funding}

This research did not receive any specific grant from funding agencies in the public, commercial, or not-for-profit sectors.

\section{Availability of data and materials}

All data generated or analyzed during this study are included in this published article [and its supplementary information files].

\section{Declarations}

\section{Ethics approval and consent to participate}

This is a retrospective study, and the need of informed consent was waived by the Medical Ethics Committee of The Fifth Affiliated Hospital Sun Yat-Sen University.

\section{Consent for publication}

Not applicable.

\section{Competing interests}

The authors declare that they have no competing interests.

\section{Author details}

${ }^{1}$ Department of Endocrinology and Metabolism, The Fifth Affiliated Hospital Sun Yat-Sen University, Zhuhai, Guangdong, China. ${ }^{2}$ Department of Health Management Center, The Fifth Affiliated Hospital Sun Yat-Sen University, Zhuhai, Guangdong, China. ${ }^{3}$ Key Laboratory of Biomedical Imaging of Guangdong Province, Guangdong Provincial Engineering Research Center of Molecular Imaging, The Fifth Affiliated Hospital Sun Yat-sen University, Zhuhai, Guangdong, China. ${ }^{4}$ Center for Interventional Medicine, The Fifth Affiliated Hospital Sun Yat-Sen University, Zhuhai, Guangdong, China.
Received: 15 November 2020 Accepted: 6 April 2021 Published online: 08 May 2021

\section{References}

1. Weekly operational update on COVID-19 - 8 March 2021, 2021.

2. Leigh R, Proud D. Virus-induced modulation of lower airway diseases: pathogenesis and pharmacologic approaches to treatment. Pharmacol Ther. 2015;148:185-98.

3. Akaike T, Suga M, Maeda H. Free radicals in viral pathogenesis: molecular mechanisms involving superoxide and NO. Proc Soc Exp Biol Med. 1998; 217:64-73.

4. Papi A, Contoli M, Gasparini $P$, et al. Role of xanthine oxidase activation and reduced glutathione depletion in rhinovirus induction of inflammation in respiratory epithelial cells. J Biol Chem. 2008;283:28595-606.

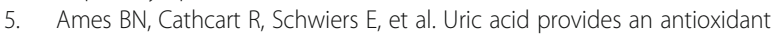
defense in humans against oxidant- and radical-caused aging and cancer: a hypothesis. Proc Natl Acad Sci U S A. 1981;78:6858-62.

6. Waring WS, Webb DJ, Maxwell SR. Systemic uric acid administration increases serum antioxidant capacity in healthy volunteers. J Cardiovasc Pharmacol. 2001;38:365-71.

7. Waring WS, McKnight JA, Webb DJ, et al. Uric acid restores endothelial function in patients with type 1 diabetes and regular smokers. Diabetes. 2006:55:3127-32.

8. Su Z, Chen Z, Xiang Y, Wang B, Huang Y, Yang D, et al. Low serum levels of uric acid and albumin in patients with Guillain-Barre syndrome. Medicine (Baltimore). 2017;96(15):e6618. https://doi.org/10.1097/MD.000000000000661 8.

9. Li S, Shao M, Tang B, et al. The association between serum uric acid and glaucoma severity in primary angle closure glaucoma: a retrospective casecontrol study. Oncotarget. 2017;8:2816-24.

10. Kim SY, Guevara JP, Kim KM, et al. Hyperuricemia and risk of stroke: a systematic review and meta-analysis. Arthritis Rheum. 2009;61:885-92.

11. Gaffo AL, Edwards NL, Saag KG. Gout. Hyperuricemia and cardiovascular disease: how strong is the evidence for a causal link? Arthritis Res Ther. 2009;11:240.

12. Sluijs I, Beulens JW, van der D.L. A, et al. Plasma uric acid is associated with increased risk of type 2 diabetes independent of diet and metabolic risk factors. J Nutr. 2013;143:80-5.

13. Johnson RJ, Perez-Pozo SE, Sautin YY, et al. Hypothesis: could excessive fructose intake and uric Acid cause type 2 diabetes? Endocr Rev. 2009;30: 96-116

14. Virdis A, Masi S, Casiglia E, et al. Identification of the uric Acid thresholds predicting an increased Total and cardiovascular mortality over 20 years. Hypertension. 2020;75:302-8.

15. Ortiz A, Massy ZA, Fliser D, et al. Clinical usefulness of novel prognostic biomarkers in patients on hemodialysis. Nat Rev Nephrol. 2011:8:141-50.

16. Tseng WC, Chen YT, Ou SM, et al. U-shaped association between serum uric acid levels with cardiovascular and all-cause mortality in the elderly: the role of malnourishment, J AM HEART ASSOC 7; 2018.

17. Odden MC, Amadu AR, Smit E, et al. Uric acid levels, kidney function, and cardiovascular mortality in US adults: National Health and nutrition examination survey (NHANES) 1988-1994 and 1999-2002. Am J Kidney Dis. 2014;64:550-7.

18. Kobylecki CJ, Afzal S, Nordestgaard BG. Plasma Urate, cancer incidence, and all-cause mortality: a Mendelian randomization study. Clin Chem. 2017;63: $1151-60$. 
19. Schuler CT, Malinczak CA, Best S, et al. Inhibition of uric acid or IL-1 beta ameliorates respiratory syncytial virus immunopathology and development of asthma. Allergy. 2020;75(9):2279-93. https://doi.org/10.1111/all.14310.

20. Liu J, Li M, Wang $X$, et al. Serum uric acid levels in patients with infections of central nervous system. Acta Neurol Belg. 2016;116:303-8.

21. Wang Y, Zhu F, Wang C, Wu J, Liu J, Chen X, Xiao H, Liu Z, Wu Z, Lu X, Ma J, Zeng Y, Peng H, Sun D. Children Hospitalized With Severe COVID-19 in Wuhan. Pediatr Infect Dis J. 2020;39(7):e91-e94. https://doi.org/10.1097/INF. 0000000000002739

22. National Health Commission of the PRC. 2021. http://www.nhc.gov.cn/xcs/ zhengcwj/202002/3b09b894ac9b4204a79db5b8912d4440.shtml.

23. Allison SJ. Chronic kidney disease: the CKD-EPI equation--accurately stratifying risk in CKD. Nat Rev Nephrol. 2012;8:371.

24. Bowman GL, Shannon J, Frei B, et al. Uric acid as a CNS antioxidant. J Alzheimers Dis. 2010:19:1331-6.

25. Du N, Xu D, Hou X, et al. Inverse association between serum uric Acid levels and Alzheimer's disease risk. Mol Neurobiol. 2016;53:2594-9.

26. Collazos J, Blanco MS, Guerra E, et al. Sequential evaluation of serum urate concentrations in AIDS patients with infections of the central nervous system. Clin Chem Lab Med. 2000;38:1293-6.

27. Dickerhof $\mathrm{N}$, Turner R, Khalilova I, et al. Oxidized glutathione and uric acid as biomarkers of early cystic fibrosis lung disease. J Cyst Fibros. 2017;16:214-21.

28. Montero-Chacón LB, Padilla-Cuadra Jl, Chiou SH, Torrealba-Acosta G. Highdensity lipoprotein, Mean Platelet Volume, and Uric Acid as Biomarkers for Outcomes in Patients with sepsis: an observational study. J Intensive Care Med. 2020;35(7):636-42. https://doi.org/10.1177/0885066618772825. Epub 2018 May 2

29. Jing LJ, Liu J, Chen Y, et al. Characteristics of laboratory findings of COVID19 patients with comorbid diabetes mellitus. Diabetes Res Clin Pract. 2020; 167:108351.

30. Werion A, Belkhir L, Perrot M, Schmit G, Aydin S, Chen Z, et al. SARS-CoV-2 causes a specific dysfunction of the kidney proximal tubule. Kidney Int. 2020;98(5):1296-307. https://doi.org/10.1016/j.kint.2020.07.019.

31. Di WU TSXY. Plasma Metabolomic and Lipidomic alterations associated with COVID-19. Natl Sci Rev. 2020;7(7):1157-68. https://doi.org/10.1093/nsr/nwaa086.

32. Antonioli L, Pacher P, Vizi ES, et al. CD39 and CD73 in immunity and inflammation. Trends Mol Med. 2013:19:355-67.

33. Macaluso MP. Testicular factors (testosterone, hyaluronidase) and uric acid. Rev Rhum Mal Osteoartic. 1959;26:106-12.

34. Liu Y, Lin Z, Sheng C, et al. The prevalence of thyroid nodules in Northwest China and its correlation with metabolic parameters and uric acid. Oncotarget. 2017:8:41555-62.

35. Zhang X, Zhu C, Gao J, et al. Gender difference in the relationship between serum uric acid reduction and improvement in body fat distribution after laparoscopic sleeve gastrectomy in Chinese obese patients: a 6-month follow-up. Lipids Health Dis. 2018;17:288.

\section{Publisher's Note}

Springer Nature remains neutral with regard to jurisdictional claims in published maps and institutional affiliations.

Ready to submit your research? Choose BMC and benefit from:

- fast, convenient online submission

- thorough peer review by experienced researchers in your field

- rapid publication on acceptance

- support for research data, including large and complex data types

- gold Open Access which fosters wider collaboration and increased citations

- maximum visibility for your research: over $100 \mathrm{M}$ website views per year

At $\mathrm{BMC}$, research is always in progress.

Learn more biomedcentral.com/submissions 